SURFACE-WATER DISCHARGE AND
EVAPOTRANSPIRATION RATES FOR GRASS AND
BARE SOIL ALONG A REACH OF THE RIO GRANDE,
ALBUQUERQUE, NEW MEXICO, 1989-95
By Condé R. Thorn

U.S. GEOLOGICAL SURVEY

Open-File Report 95-419

Prepared in cooperation with the CITY OF ALBUQUERQUE PUBLIC WORKS DEPARTMENT

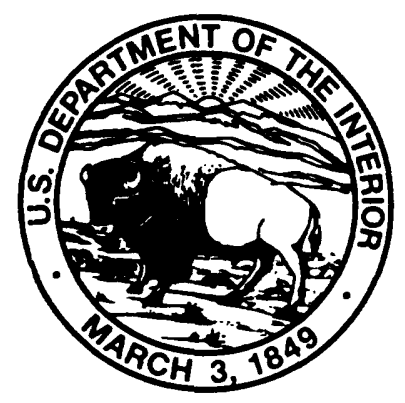

Albuquerque, New Mexico 


\section{U.S. DEPARTMENT OF THE INTERIOR \\ BRUCE BABBITT, Secretary \\ U.S. GEOLOGICAL SURVEY \\ Gordon P. Eaton, Director}

For additional information write to:

District Chief

U.S. Geological Survey

Water Resources Division

4501 Indian School Rd. NE, Suite 200

Albuquerque, New Mexico 87110-3929
Copies of this report can be purchased from:

U.S. Geological Survey Earth Science Information Center Open-File Reports Section Box 25286, MS 517 Denver Federal Center Denver, Colorado 80225 


\section{CONTENTS}

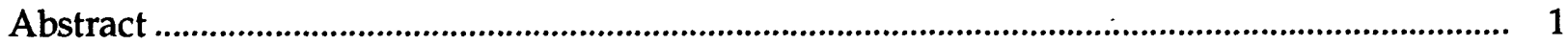



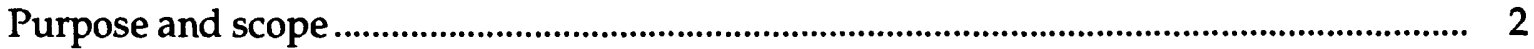

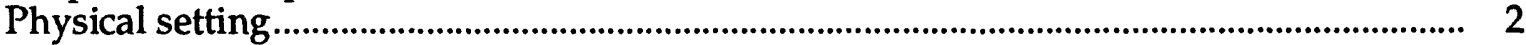

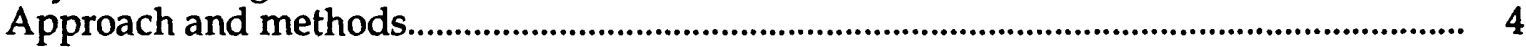

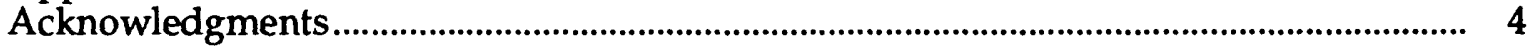

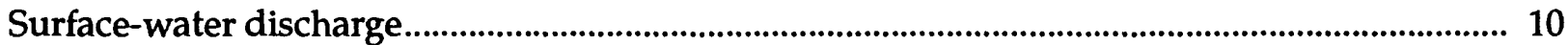

Evapotranspiration rates ................................................................................................................ 19

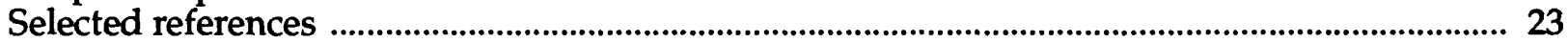

\section{FIGURES}

Figure 1. Map showing shaded relief of the Albuquerque Basin and vicinity, Central New Mexico

2. Map showing location of surface-water measurement sites and evapotranspiration measurement site in the study area

3-5. Hydrographs showing mean daily discharge of the Rio Grande at the:

3. U.S. Geological Survey gaging station Rio Grande near Alameda, New Mexico

4. U.S. Geological Survey gaging station Rio Grande at Albuquerque, New Mexico

5. U.S. Geological Survey gaging station Rio Grande at Rio Bravo Bridge near Albuquerque, New Mexico.

6. Diagrams showing Rio Grande discharge from the north section (A), south section (B), and the difference between the two (C)

7. Diagrams showing total discharge from the north section (A), south section (B), and the difference between the two (C).

8. Photographs showing hemispherical chamber located at Paseo del Norte and the Rio Grande: (A) grass covered (area 2); (B) bare soil (area 3).

9. Bar graph showing evapotranspiration rates calculated at areas 5 and 6 at Paseo del Norte and Rio Grande. 


\section{TABLES}

Table 1. Site number and name of surface-water measurement sites in the study area............. 6

2. Surface-water discharge from the Paseo del Norte (north) section.............................. 11

3. Surface-water discharge from the Rio Bravo Boulevard (south) section........................ 13

4. Surface-water discharge and differences between Rio Grande discharge and total discharge from the Paseo del Norte (north) section and the Rio Bravo Boulevard (south) section

5. Evapotranspiration rates at six areas at Paseo del Norte and the Rio

\section{CONVERSION FACTORS}

\section{Multiply}

inch

foot

mile

square mile

cubic foot per second
By

25.4

0.3048

1.609

2.590

28.32
To obtain

millimeter

meter

kilometer square kilometer liter per second

Sea level: In this report "sea level" refers to the National Geodetic Vertical Datum of 1929-a geodetic datum derived from a general adjustment of the first-order level nets of the United States and Canada, formerly called Sea Level Datum of 1929. 


\title{
SURFACE-WATER DISCHARGE AND \\ EVAPOTRANSPIRATION RATES FOR GRASS AND BARE SOIL ALONG A \\ REACH OF THE RIO GRANDE, ALBUQUERQUE, NEW MEXICO, 1989-95 \\ By Condé R. Thorn
}

\begin{abstract}
The surface-water hydrologic system of the Rio Grande in the Albuquerque, New Mexico, area is composed of the Rio Grande; irrigation drains, laterals, canals, and ditches; evapotranspiration; and ground-water recharge. Two east-west cross sections were established to measure surface water entering the study area (north section) and exiting the study area (south section). Data for the calculation of surface-water discharge were collected once every 4 to 8 weeks from August 1989 through February 1995. Discharge of the Rio Grande at the north section ranged from 22 to 5,900 cubic feet per second. Discharge of the Rio Grande at the south section ranged from 21 to 6,060 cubic feet per second.

Data from six areas (three grass-covered areas and three bare-soil areas) were collected for the calculation of evapotranspiration rates from February 1990 through August 1991. The evapotranspiration rate from the grass-covered areas ranged from 0.15 to 4.7 millimeters per day; the evapotranspiration rate from the bare-soil areas ranged from 0.13 to 6.4 millimeters per day.
\end{abstract}




\section{INTRODUCTION}

The surface-water hydrologic system within the Rio Grande flood plain in the Albuquerque, New Mexico, area has undergone many changes through the years. The Middle Rio Grande Conservancy District (MRGCD) was created in 1925 to control floods and lower the water table to prevent waterlogging of irrigated lands along the valley. The MRGCD constructed hundreds of miles of interior and riverside drains, levees, and irrigation diversion dams. The Albuquerque Metropolitan Arroyo Flood Control Authority (AMAFCA), created in 1963, constructed several detention dams and conveyance channels on the terraces and alluvial fans that the City of Albuquerque has developed (Crawford and others, 1993). These structures constructed by the MRGCD and AMAFCA allow for runoff to be either directly or indirectly discharged into the Rio Grande and have complicated the interaction between the surface-water and ground-water systems. Further complicating this interaction between the surface-water and ground-water systems is the conversion of irrigated lands to urban development. A greater understanding of the surface-water hydrologic system needs to be established so that the City of Albuquerque can develop the most efficient and productive management of its water resources.

\section{Purpose and Scope}

This report presents a summary of surface-water discharge from the major surface-water components in the flood plain along a reach of the Rio Grande in the Albuquerque, New Mexico, area. Data used for the calculation of surface-water discharge were collected from August 1989 through February 1995. Also contained in this report are evapotranspiration rates calculated from three grass-covered areas and three bare-soil areas on the Rio Grande. Evapotranspiration rates are presented for 14 months from February 1990 through August 1991.

\section{Physical Setting}

The Albuquerque Basin, located in central New Mexico (fig. 1), covers approximately 3,060 square miles. The land-surface altitude in the basin ranges from about 6,500 feet above sea level in the northern part of the basin to about 4,800 feet in the southern part of the basin. The eastern boundary of the basin has the greatest topographic relief and is defined by the Sandia, Manzanita, Manzano, and Los Pinos Mountains. The western boundary has little topographic relief and is defined by Mesa Lucero, Sierra Lucero, and Ladron Peak. The convergence of the structural eastern and western boundaries forms the northern and southern boundaries.

The Rio Grande and its associated flood-plain deposits trend in a north-to-south direction in the central part of the Albuquerque Basin. The city of Albuquerque is on the east and west banks of the Rio Grande within the central part of the basin. Directly east of Albuquerque the Sandia Mountains rise to an altitude greater than 10,000 feet above sea level. West of Albuquerque the topography has an average altitude of more than 5,000 feet and is characterized as generally flat with several volcanic features and dune fields (Kernodle and others, 1987). 


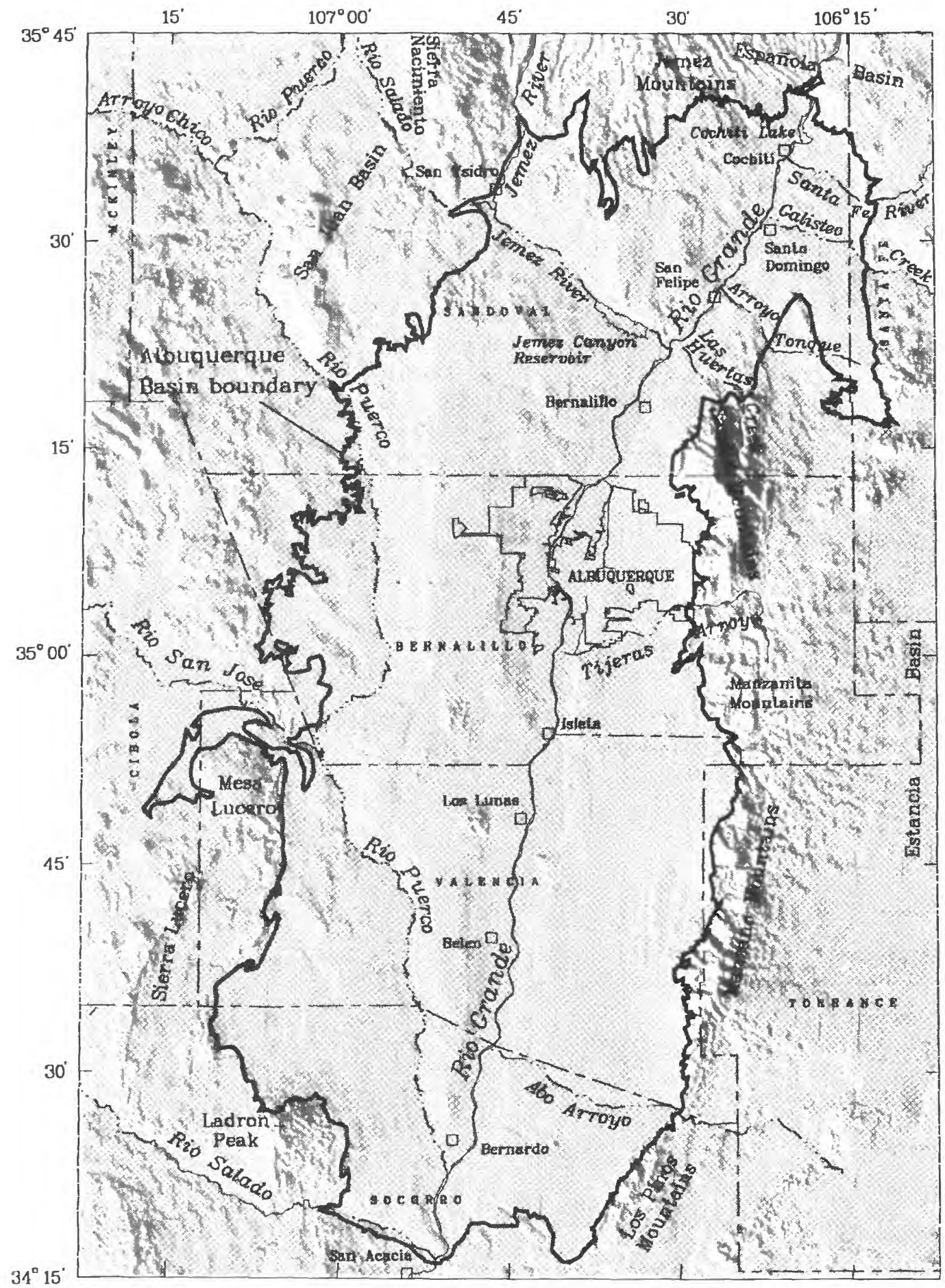

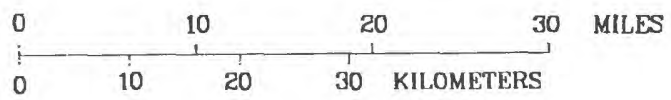

Figure 1.--Shaded relief of the Albuquerque Basin and vicinity, Central New Mexico (from Thorn and others, 1993). 


\section{Approach and Methods}

Two east-west cross sections, located at the northern and southern boundaries of the study area, were established to make instantaneous surface-water measurements for the calculation of surface water discharge entering and exiting the study area. The northern boundary (north section) is an east-west line through the intersection of the Rio Grande with Paseo del Norte (fig. 2; table 1). An east-west line through the intersection of the Rio Grande with Rio Bravo Boulevard is the southern boundary (south section). Surface-water measurements were made within a 10-hour period every 4 to 8 weeks at all measurement sites along the northern and southern boundaries of the study area. The day selected to make the surface-water measurements was based on the stability of flow in the Rio Grande--that is, about 48 hours would elapse after a release of water from a major reservoir upstream from the study area and after any measurable precipitation within the Albuquerque area. All surface-water measurements were made by U.S. Geological Survey personnel using current meters as outlined in Buchanan and Somers (1984), Kennedy (1984), and Carter and Davidian (1989). In addition to the instantaneous surface-water measurements made, continuous records of streamflow were available from three U.S. Geological Survey gaging stations on the Rio Grande in the study area (figs. 2-5).

The rate of evapotranspiration from three grass-covered and three bare-soil areas was calculated using a hemispherical chamber. The location of the site of the six evapotranspiration areas is shown in figure 2; distance between the individual areas varies from 100 to 150 feet. Descriptions of the six areas and the collection of data for the calculation of evapotranspiration rates are discussed in a subsequent section of this report.

\section{Acknowledgments}

The author extends his appreciation to all the hydrologic technicians from the U.S. Geological Survey Albuquerque field office that performed the surface-water measurements on a regular frequency. This report would not have been possible without their commitment to regular visits to the 23 sites over a $41 / 2$-year period. 


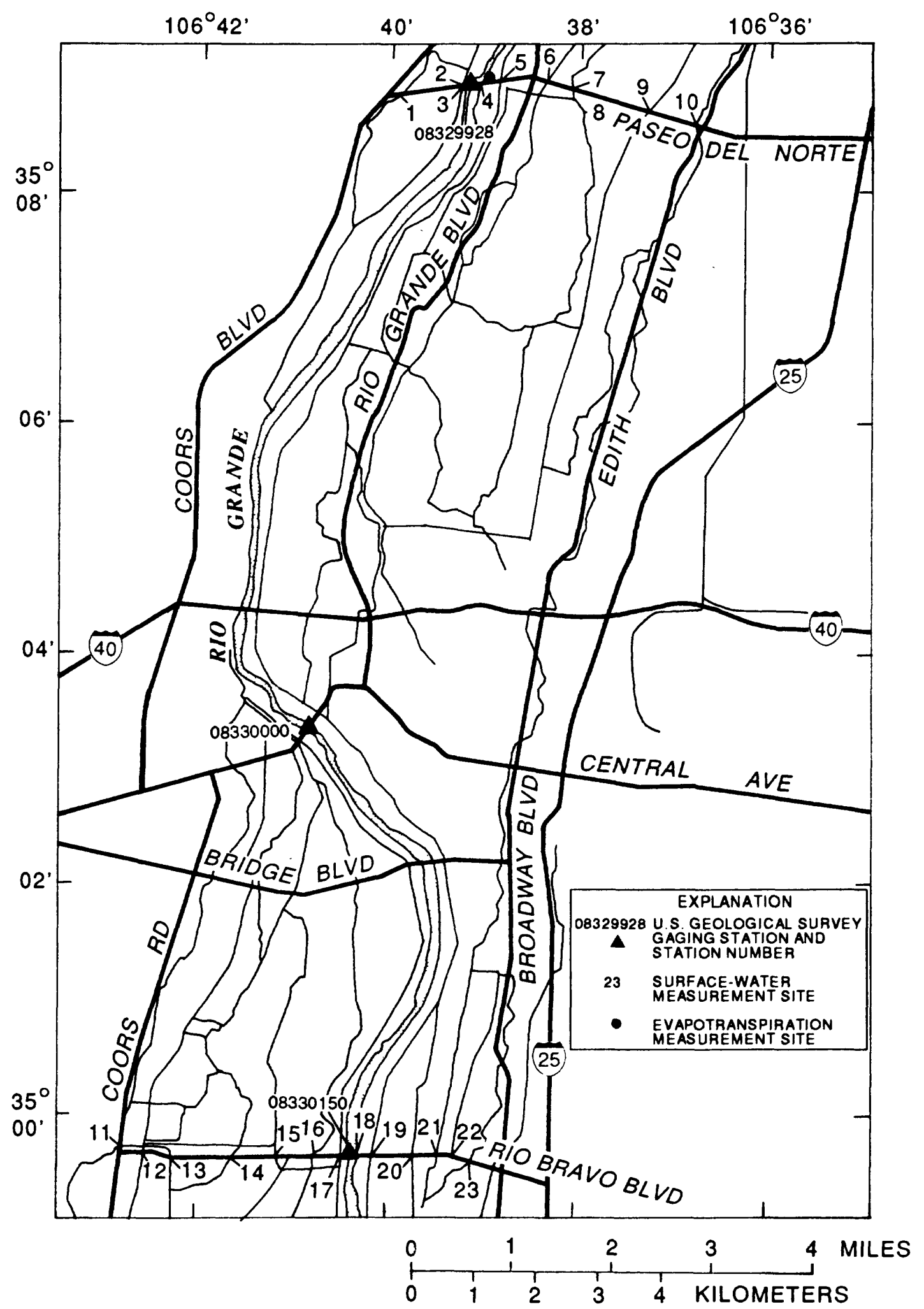

Figure 2.--Location of surface-water measurement sites and evapotranspiration measurement site in the study area. See table 1 for names of surface-water measurement sites. 
Table 1.--Site number and name of surface-water measurement sites in the study area [See figure 2 for location of sites]

\begin{tabular}{|c|c|c|c|}
\hline \multicolumn{4}{|c|}{ Surface-water measurement site number and name } \\
\hline \multicolumn{2}{|r|}{ North section } & \multicolumn{2}{|r|}{ South section } \\
\hline 1 & Corrales Main Canal & 11 & Gun Club Lateral \\
\hline 2 & Ditch & 12 & Isleta Drain \\
\hline 3 & Corrales Riverside Drain & 13 & Arenal Main Canal \\
\hline 4 & Rio Grande at Paseo del Norte & 14 & Pajarito Lateral \\
\hline 5 & Albuquerque Riverside Drain & 15 & Beckman Lateral \\
\hline 6 & Albuquerque Main Canal & 16 & Atrisco Drain \\
\hline 7 & Chamizal Lateral & 17 & Atrisco Riverside Drain \\
\hline 8 & Derromadera Ditch & 18 & Rio Grande at Rio Bravo Boulevard \\
\hline 9 & Alameda Drain & 19 & Albuquerque Riverside Drain \\
\hline \multirow[t]{4}{*}{10} & Alameda Lateral & 20 & Barr Canal \\
\hline & & 21 & Barelas Ditch \\
\hline & & 22 & San Jose Drain \\
\hline & & 23 & San Jose Lateral \\
\hline
\end{tabular}




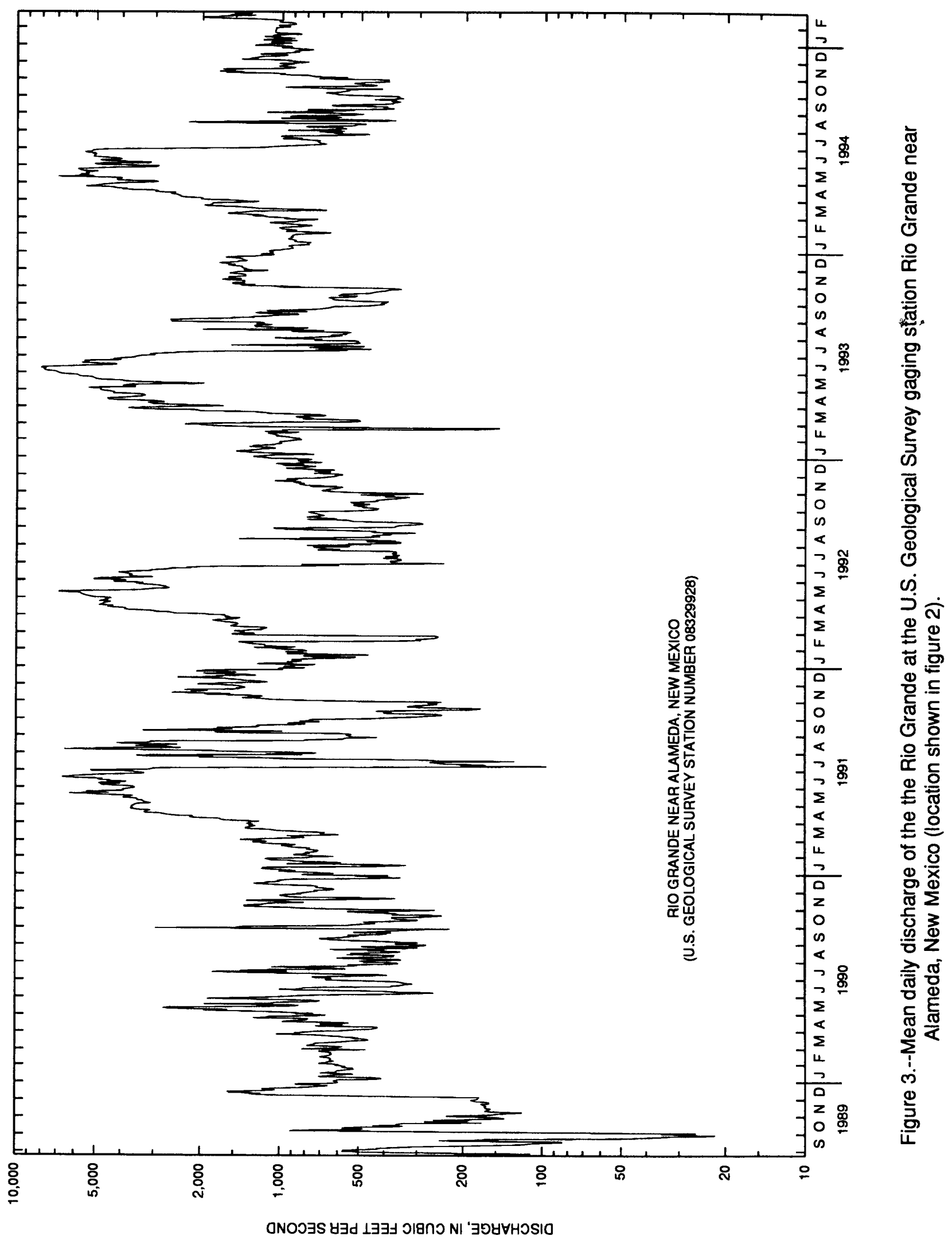




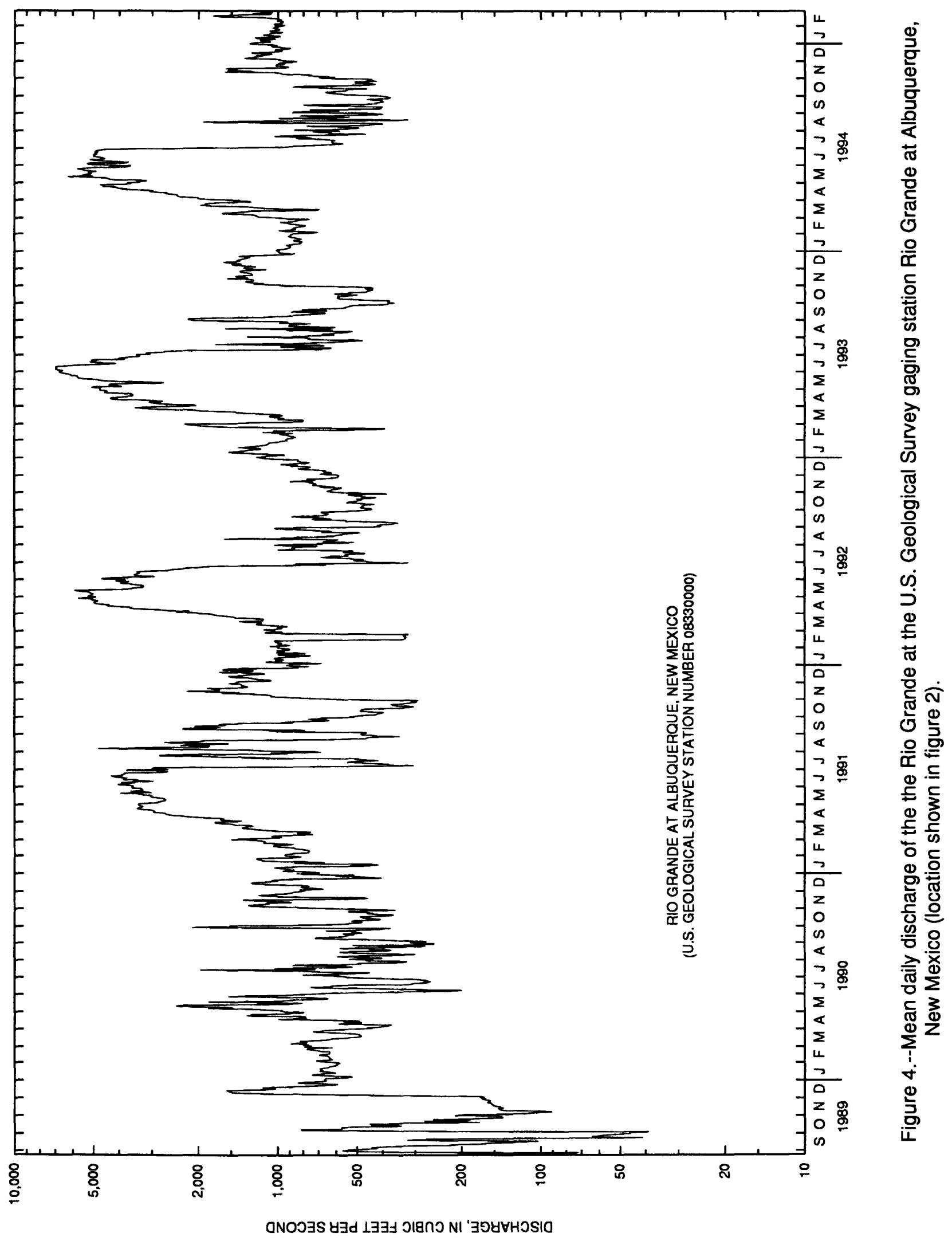









\section{SURFACE-WATER DISCHARGE}

Instantaneous surface-water measurements were made of the Rio Grande and all irrigation drains, laterals, canals, and ditches along the north and south sections. The north section had 10 measurement sites and the south section had 13 measurement sites (fig. 2; table 1). The discharge was calculated for each site, and the sum of discharge for all sites along both the north and south sections represents the total surface-water discharge entering and exiting the study area, respectively (tables 2 and 3 ).

Rio Grande discharge and total surface-water discharge (total discharge) are shown in table 4 and figures 6 and 7. Discharge of the Rio Grande at the north section ranged from 22 to 5,900 cubic feet per second, and at the south section ranged from 21 to 6,060 cubic feet per second (table 4). Also shown in table 4 and figures 6 and 7 are the differences in discharge between the north and south sections for both Rio Grande discharge and total discharge. Rio Grande discharge entering the study area was greater than Rio Grande discharge exiting the study area (negative difference) on 45 of 61 days; Rio Grande discharge entering the study area was less than that exiting the study area (positive difference) on 16 of the 61 days (table 4; figs. 6 and 7). Total discharge entering the study area was greater than that exiting the study area on 48 of the 59 days shown in table 4 and figures 6 and 7; 11 of the 59 days had lower total discharge entering the study area than total discharge exiting the study area. The total number of measurements of recorded Rio Grande discharge (61) differs from the total number of measurements of recorded total discharge (59) because on two different occasions (September 26, 1991, and February 19, 1992) no measurement was made at one of the sites, therefore a true total discharge could not be calculated (table 4). 
Table 2.--Surface-water discharge from the Paseo del Norte (north) section

[See figure 2 for location and table 1 for name of sites. Values are in cubic feet per second; totals are rounded; - -, no data]

\begin{tabular}{|c|c|c|c|c|c|c|c|c|c|c|c|}
\hline Date & Site 1 & Site 2 & Site 3 & Site 4 & Site 5 & Site 6 & Site 7 & Site 8 & Site 9 & Site 10 & Total \\
\hline $08 / 25 / 89$ & 27 & 6.6 & 17 & 251 & 156 & 129 & 9.2 & 0 & 0.9 & 4.2 & 600 \\
\hline $09 / 29 / 89$ & 22 & 8 & 13 & 22 & 146 & 91 & 16 & 2.8 & 0 & 0 & 320 \\
\hline $10 / 27 / 89$ & 15 & 4 & 4.4 & 115 & 130 & 126 & 8.9 & 2 & 3.8 & 1.1 & 410 \\
\hline $12 / 01 / 89$ & 0 & 0 & 4.2 & 171 & 212 & 0 & 0 & 0 & 0 & 0 & 390 \\
\hline $12 / 29 / 89$ & 0 & 0 & 5 & 757 & 58 & 0 & 0 & 0 & 0 & 0 & 820 \\
\hline $01 / 31 / 90$ & 0 & 0 & 4.8 & 518 & 56 & 0 & 0 & 0 & 0 & 0 & 580 \\
\hline $02 / 28 / 90$ & 0 & 0 & 3.8 & 292 & 48 & 0 & 0 & 0 & 0 & 0 & 340 \\
\hline $03 / 30 / 90$ & 20 & 0 & 16 & 875 & 154 & 85 & 11 & 0.3 & 5 & 12 & 1,180 \\
\hline $04 / 30 / 90$ & 12 & 2.5 & 9.2 & 681 & 180 & 121 & 11 & 0.9 & 4.7 & 13 & 1,030 \\
\hline $05 / 31 / 90$ & 22 & 0.6 & 11 & 1,190 & 214 & 136 & 8.8 & 0.5 & 8.8 & 6.8 & 1,600 \\
\hline $06 / 29 / 90$ & 11 & 1.1 & 8 & 428 & 147 & 116 & 7.5 & 2.3 & 5.2 & 3.3 & 730 \\
\hline $07 / 31 / 90$ & 0.7 & 5 & 10 & 398 & 168 & 120 & 5.6 & 0 & 2.1 & 11 & 720 \\
\hline $08 / 30 / 90$ & 16 & 7.7 & 15 & 285 & 162 & 118 & 13 & 1.3 & 0.8 & 2.7 & 620 \\
\hline $09 / 27 / 90$ & 22 & 1.6 & 12 & 260 & 212 & 127 & 14 & 1.6 & 3.8 & 7.2 & 660 \\
\hline $10 / 30 / 90$ & 6.5 & 3.8 & 13 & 307 & 164 & 117 & 12 & 1.1 & 1.8 & 6 & 630 \\
\hline $12 / 11 / 90$ & 0 & 0 & 4.6 & 699 & 70 & 0 & 0 & 0 & 0 & 0 & 770 \\
\hline $01 / 10 / 91$ & 0 & 0 & 4.2 & 1,040 & 52 & 0 & 0 & 0 & 0 & 0 & 1,100 \\
\hline $01 / 31 / 91$ & 0 & 0 & 4.1 & 742 & 64 & 0 & 0 & 0 & 0 & 0 & 810 \\
\hline $02 / 21 / 91$ & 0 & 0 & 4 & 869 & 59 & 0 & 0 & 0 & 0 & 0 & 930 \\
\hline $03 / 27 / 91$ & 9.8 & 0.2 & 5 & 1,290 & 155 & 131 & 15 & 0 & 2.6 & 0 & 1,610 \\
\hline $05 / 02 / 91$ & 15 & 9.4 & 9.2 & 3,580 & 227 & 115 & 15 & 0.4 & 6.2 & 6.1 & 3,980 \\
\hline $05 / 30 / 91$ & 19 & 4.4 & 8.6 & 3,920 & 178 & 176 & 15 & 0 & 13 & 9.6 & 4,340 \\
\hline $06 / 28 / 91$ & 18 & 4.3 & 9.2 & 3,490 & 184 & 120 & 7.1 & 2.3 & 4.2 & 1.3 & 3,840 \\
\hline $07 / 31 / 91$ & 13 & 15 & 6.4 & 953 & 166 & 145 & 26 & 8.6 & 6 & 7.1 & 1,350 \\
\hline $08 / 29 / 91$ & 15 & 21 & 8 & 399 & 144 & 158 & 18 & 0 & 7.9 & 0 & 770 \\
\hline $09 / 26 / 91$ & 8.4 & 23 & 5.6 & 645 & 169 & -- & 13 & 2.9 & 1.7 & 1.1 & -- \\
\hline $10 / 31 / 91$ & 10 & 15 & 4.8 & 320 & 145 & 132 & 23 & 1.6 & 2.4 & 4.4 & 660 \\
\hline $11 / 27 / 91$ & 0 & 0 & 6.8 & 1,430 & 53 & 0 & 0 & 0 & 0 & 0 & 1,490 \\
\hline $12 / 31 / 91$ & 0 & 0 & 5.2 & 1,050 & 55 & 0 & 0 & 0 & 0 & 0 & 1,110 \\
\hline $01 / 30 / 92$ & 0 & 0 & 4.2 & 859 & 52 & 0 & 0 & 0 & 0 & 0 & 910 \\
\hline $02 / 19 / 92$ & 0 & 0 & 3.7 & 306 & 49 & 0 & 0 & 0 & 0 & 0 & 360 \\
\hline $04 / 08 / 92$ & 1.2 & 21 & 7.8 & 2,890 & 163 & 108 & 17 & 1.2 & 6.1 & 0 & 3,210 \\
\hline $04 / 30 / 92$ & 1.8 & 9.3 & 7.4 & 5,560 & 179 & 135 & 18 & 3.3 & 2.8 & 5.7 & 5,920 \\
\hline $06 / 30 / 92$ & 5 & 11 & 6.2 & 912 & 187 & 84 & 11 & 0.7 & 0.9 & 0.1 & 1,220 \\
\hline $07 / 31 / 92$ & 17 & 2.6 & 4.3 & 734 & 177 & 163 & 18 & 3.9 & 4.8 & 0 & 1,120 \\
\hline $08 / 31 / 92$ & 23 & 1.7 & 4.2 & 1,120 & 182 & 130 & 17 & 2.3 & 2.2 & 11 & 1,490 \\
\hline $09 / 30 / 92$ & 6.8 & 29 & 2 & 566 & 173 & 125 & 19 & 3.9 & 6.5 & 5.3 & 940 \\
\hline
\end{tabular}


Table 2.--Surface-water discharge from the Paseo del Norte (north) section--Concluded

\begin{tabular}{|c|c|c|c|c|c|c|c|c|c|c|c|}
\hline Date & Site 1 & Site 2 & Site 3 & Site 4 & Site 5 & Site 6 & Site 7 & Site 8 & Site 9 & Site 10 & Tota \\
\hline $11 / 02 / 92$ & 0 & 0 & 3.4 & 608 & 119 & 0.6 & 0 & 0 & 0 & 0 & 730 \\
\hline $11 / 30 / 92$ & 0 & 0 & 4.1 & 614 & 61 & 0 & 0 & 0 & 0 & 0 & 680 \\
\hline $01 / 04 / 93$ & 0 & 0 & 4.4 & 1,160 & 54 & 0 & 0 & 0 & 0 & 0 & 1,220 \\
\hline $01 / 28 / 93$ & 0 & 0 & 3.9 & 1,030 & 82 & 0 & 0 & 0 & 0 & 0 & 1,120 \\
\hline $02 / 26 / 93$ & 0 & 0 & 4.3 & 1,940 & 62 & 0 & 0 & 0 & 0 & 0 & 2,010 \\
\hline $03 / 31 / 93$ & 19 & 0 & 13 & 2,840 & 145 & 115 & 15 & 0 & 2.6 & 4.4 & 3,150 \\
\hline $04 / 30 / 93$ & 0 & 9.9 & 7.9 & 4,880 & 147 & 98 & 12 & 1.4 & 2.9 & 10 & 5,170 \\
\hline $05 / 27 / 93$ & 15 & 13 & 10 & 5,900 & 177 & 170 & 21 & 2.2 & 3.5 & 2 & 6,310 \\
\hline $06 / 30 / 93$ & 14 & 2.3 & 6.2 & 3,550 & 211 & 120 & 13 & 0 & 3.1 & 0 & 3,920 \\
\hline $07 / 30 / 93$ & 13 & 1.7 & 5.3 & 811 & 143 & 104 & 19 & 3.6 & 4 & 8.6 & 1,110 \\
\hline $09 / 30 / 93$ & 9.7 & 13 & 2.4 & 438 & 130 & 104 & 12 & 2.4 & 9 & 9.4 & 730 \\
\hline $10 / 22 / 93$ & 0 & 25 & 2.6 & 450 & 137 & 98 & 16 & 1.4 & 1.8 & 11 & 740 \\
\hline $12 / 06 / 93$ & 0 & 0 & 3 & 1,420 & 67 & 0 & 0 & 0 & 0 & 0 & 1,490 \\
\hline $01 / 28 / 94$ & 0 & 0 & 1.8 & 943 & 58 & 0 & 0 & 0 & 0 & 0 & 1,000 \\
\hline $02 / 28 / 94$ & 0 & 0 & 2.3 & 1,040 & 50 & 0 & 0 & 0 & 0 & 0 & 1,090 \\
\hline 04/01/94 & 17 & 0 & 3.1 & 1,470 & 155 & 113 & 14 & 0.8 & 4.8 & 9.4 & 1,790 \\
\hline $05 / 02 / 94$ & 17 & 0 & 3.1 & 3,230 & 186 & 110 & 15 & 2.5 & 3.5 & 9.1 & 3,580 \\
\hline $06 / 27 / 94$ & 20 & 0 & 3.6 & 5,300 & 189 & 94 & 10 & 4.2 & 2.3 & 4 & 5,630 \\
\hline $08 / 29 / 94$ & 14 & 0 & 1.8 & 510 & 154 & 131 & 4.4 & 3 & 1.5 & 8.9 & 830 \\
\hline $09 / 30 / 94$ & 28 & 0 & 2.1 & 375 & 138 & 187 & 18 & 3 & 0.8 & 4.6 & 760 \\
\hline $10 / 31 / 94$ & 19 & 0 & 2 & 397 & 158 & 108 & 13.6 & 0.4 & 0.9 & 2.3 & 700 \\
\hline $12 / 01 / 94$ & 0 & 0 & 0.3 & 783 & 75 & 0 & 0 & 0 & 0 & 0 & 860 \\
\hline $01 / 30 / 95$ & 0 & 0 & 2.6 & 1,040 & 50 & 0 & 0 & 0 & 0 & 0 & 1,090 \\
\hline $02 / 28 / 95$ & 0 & 0 & 2.1 & 1,610 & 57 & 0 & 0 & 0 & 0 & 0 & 1,670 \\
\hline
\end{tabular}


[See figure 2 for location and table 1 for names of sites. Values are in cubic feet per second; totals are rounded; --, no data]

\begin{tabular}{|c|c|c|c|c|c|c|c|c|c|c|c|c|c|c|}
\hline Date & $\begin{array}{c}\text { Site } \\
11\end{array}$ & $\begin{array}{c}\text { Site } \\
12\end{array}$ & $\begin{array}{c}\text { Site } \\
13\end{array}$ & $\begin{array}{c}\text { Site } \\
14\end{array}$ & $\begin{array}{c}\text { Site } \\
15\end{array}$ & $\begin{array}{c}\text { Site } \\
16\end{array}$ & $\begin{array}{c}\text { Site } \\
17\end{array}$ & $\begin{array}{c}\text { Site } \\
18\end{array}$ & $\begin{array}{c}\text { Site } \\
19\end{array}$ & $\begin{array}{c}\text { Site } \\
20\end{array}$ & $\begin{array}{c}\text { Site } \\
21\end{array}$ & $\begin{array}{c}\text { Site } \\
22\end{array}$ & $\begin{array}{c}\text { Site } \\
23\end{array}$ & Total \\
\hline $08 / 25 / 89$ & 17 & 8.7 & 65 & 0 & 28 & 11 & 30 & 243 & 70 & 56 & 0 & 0 & 0 & 530 \\
\hline $09 / 29 / 89$ & 0 & 9.2 & 25 & 11 & 19 & 10 & 29 & 21 & 54 & 60 & 0 & 0 & 0 & 240 \\
\hline $10 / 27 / 89$ & 11 & 6.1 & 28 & 1.5 & 24 & 7.9 & 20 & 112 & 42 & 45 & 0 & 0 & 0 & 300 \\
\hline $12 / 01 / 89$ & 0 & 0 & 0 & 0 & 0 & 0 & 60 & 131 & 170 & 0 & 0 & 0 & 0 & 360 \\
\hline $12 / 29 / 89$ & 0 & 0 & 0 & 0 & 0 & 0 & 17 & 729 & 27 & 0 & 0 & 0 & 0 & 770 \\
\hline $01 / 31 / 90$ & 0 & 0 & 0 & 0 & 0 & 0 & 18 & 511 & 27 & 0 & 0 & 0 & 0 & 560 \\
\hline $02 / 28 / 90$ & 0 & 0 & 0 & 0 & 0 & 0 & 18 & 545 & 29 & 0 & 0 & 0 & 0 & 590 \\
\hline $03 / 30 / 90$ & 29 & 11 & 19 & 15 & 36 & 13 & 26 & 715 & 107 & 51 & 0 & 0 & 0 & 1,020 \\
\hline $04 / 30 / 90$ & 3.4 & 4 & 37 & 0.1 & 25 & 1.9 & 21 & 735 & 92 & 12 & 0 & 0 & 0 & 930 \\
\hline $05 / 31 / 90$ & 17 & 4.9 & 43 & 5.9 & 33 & 13 & 29 & 1,020 & 100 & 66 & 0 & 0 & 0 & 1,330 \\
\hline $06 / 29 / 90$ & 6.6 & 6.9 & 55 & 11 & 12 & 8.9 & 22 & 313 & 93 & 50 & 0 & 0 & 0 & 580 \\
\hline $07 / 31 / 90$ & 8.4 & 9 & 44 & 18 & 27 & 4.4 & 36 & 269 & 71 & 52 & 0 & 0 & 0 & 540 \\
\hline $08 / 30 / 90$ & 17 & 6.5 & 43 & 3.5 & 30 & 8.8 & 35 & 183 & 108 & 72 & 0 & 0 & 0 & 510 \\
\hline $09 / 27 / 90$ & 2.4 & 7.8 & 40 & 10 & 13 & 14 & 33 & 296 & 97 & 60 & 0 & 0 & 0 & 570 \\
\hline $10 / 30 / 90$ & 11 & 4.7 & 22 & 2.6 & 22 & 10 & 33 & 360 & 77 & 42 & 0 & 0 & 0 & 580 \\
\hline $12 / 11 / 90$ & 0 & 0.8 & 0 & 0 & 0 & 0 & 25 & 652 & 29 & 0 & 0 & 0 & 0 & 710 \\
\hline $01 / 10 / 91$ & 0 & 0.6 & 0 & 0 & 0 & 0 & 25 & 1,060 & 29 & 0 & 0 & 0 & 0 & 1,110 \\
\hline $01 / 31 / 91$ & 0 & 0.5 & 0 & 0 & 0 & 0 & 20 & 737 & 27 & 0 & 0 & 0 & 0 & 780 \\
\hline $02 / 21 / 91$ & 0 & 0 & 0 & 0 & 0 & 0 & 23 & 898 & 26 & 0 & 0 & 0 & 0 & 950 \\
\hline $03 / 27 / 91$ & 0 & 1.4 & 32 & 6.3 & 11 & 4.4 & 35 & 1,450 & 74 & 64 & 0 & 0 & 0 & 1,680 \\
\hline $05 / 02 / 91$ & 14 & 8.9 & 36 & 9.9 & 19 & 6.2 & 31 & 2,970 & 99 & 54 & 0 & 0 & 0 & 3,250 \\
\hline $05 / 30 / 91$ & 19 & 7.5 & 49 & 9 & 19 & 7.2 & 42 & 4,090 & 139 & 63 & 0 & 0 & 0 & 4,440 \\
\hline $06 / 28 / 91$ & 21 & 13 & 51 & 5.2 & 25 & 4.3 & 60 & 3,050 & 78 & 59 & 0 & 0 & 0 & 3,370 \\
\hline $07 / 31 / 91$ & 0 & 6.1 & 44 & 0 & 26 & 9.4 & 41 & 1,150 & 178 & 65 & 0 & 0 & 0 & 1,520 \\
\hline $08 / 29 / 91$ & 15 & 6.4 & 42 & 8.1 & 34 & 9.4 & 31 & 270 & 101 & 63 & 0 & 0 & 0 & 580 \\
\hline $09 / 26 / 91$ & 5 & 12 & 27 & 12 & 19 & 10 & 36 & 600 & 84 & 34 & 0 & 0 & 0 & 840 \\
\hline $10 / 31 / 91$ & 3.2 & 22 & 19 & 9 & 28 & 2.6 & 37 & 267 & 134 & 39 & 0 & 0 & 0 & 560 \\
\hline $11 / 27 / 91$ & 0 & 1.6 & 0 & 0 & 0 & 0 & 32 & 1,270 & 28 & 0 & 0 & 0 & 0 & 1,330 \\
\hline $12 / 31 / 91$ & 0 & 1.2 & 0 & 0 & 0 & 0 & 23 & 913 & 27 & 0 & 0 & 0 & 0 & 960 \\
\hline $01 / 30 / 92$ & 0 & 0.8 & 0 & 0 & 0 & 0 & 24 & 824 & 27 & 0 & 0 & 0 & 0 & 880 \\
\hline $02 / 19 / 92$ & 0 & 0.5 & -. & 0 & 0 & 0 & 22 & 338 & 23 & 0 & 0 & 0 & 0 & $\cdots$ \\
\hline $04 / 08 / 92$ & 19 & 8.6 & 42 & 6.6 & 29 & 6.8 & 37 & 2,640 & 96 & 71 & 0 & 0 & 0 & 2,960 \\
\hline $04 / 30 / 92$ & 17 & 9.2 & 48 & 1.8 & 34 & 6.9 & 52 & 5,120 & 112 & 67 & 0 & 0 & 0 & 5,470 \\
\hline $06 / 30 / 92$ & 11 & 9.5 & 56 & 4.1 & 22 & 6.2 & 31 & 815 & 79 & 56 & 0 & 0 & 0 & 1,090 \\
\hline $07 / 31 / 92$ & 7.4 & 8.7 & 28 & 4.4 & 37 & 11 & 41 & 687 & 101 & 89 & 0 & 0 & 0 & 1,010 \\
\hline $08 / 31 / 92$ & 14 & 8.6 & 41 & 6.1 & 27 & 6.2 & 31 & 1,260 & 96 & 61 & 0 & 0 & 0 & 1,550 \\
\hline $09 / 30 / 92$ & 10 & 11 & 42 & 6 & 32 & 3.8 & 35 & 543 & 96 & 26 & 0 & 0 & 0 & 800 \\
\hline $11 / 02 / 92$ & 0 & 9.3 & 4.2 & 1.1 & 0.1 & 0 & 28 & 501 & 55 & 0 & 0 & 0 & 0 & 600 \\
\hline $11 / 30 / 92$ & 0 & 1.1 & 0 & 0 & 0 & 0 & 22 & 690 & 21 & 0 & 0 & 0 & 0 & 730 \\
\hline $01 / 04 / 93$ & 0 & 0.2 & 0 & 0 & 0 & 0 & 24 & 1,060 & 24 & 0 & 0 & 0 & 0 & 1,110 \\
\hline $01 / 28 / 93$ & 0 & 0.4 & 0 & 0 & 0 & 0 & 21 & 1,070 & 24 & 0 & 0 & 0 & 0 & 1,110 \\
\hline $02 / 26 / 93$ & 0 & 0.1 & 0 & 0 & 0 & 0 & 21 & 2,460 & 21 & 0 & 0 & 0 & 0 & 2,500 \\
\hline $03 / 31 / 93$ & 15 & 8.1 & 27 & 11 & 30 & 13 & 37 & 2,830 & 76 & 54 & 0 & 0 & 0 & 3,100 \\
\hline
\end{tabular}


Table 3.--Surface-water discharge from the Rio Bravo Boulevard (south) section--Concluded

\begin{tabular}{|c|c|c|c|c|c|c|c|c|c|c|c|c|c|c|}
\hline Date & $\begin{array}{c}\text { Site } \\
11\end{array}$ & $\begin{array}{c}\text { Site } \\
12\end{array}$ & $\begin{array}{c}\text { Site } \\
13\end{array}$ & $\begin{array}{c}\text { Site } \\
14\end{array}$ & $\begin{array}{c}\text { Site } \\
15\end{array}$ & $\begin{array}{c}\text { Site } \\
16\end{array}$ & $\begin{array}{c}\text { Site } \\
17\end{array}$ & $\begin{array}{c}\text { Site } \\
18\end{array}$ & $\begin{array}{c}\text { Site } \\
19\end{array}$ & $\begin{array}{c}\text { Site } \\
20\end{array}$ & $\begin{array}{c}\text { Site } \\
21\end{array}$ & $\begin{array}{c}\text { Site } \\
22\end{array}$ & $\begin{array}{c}\text { Site } \\
23\end{array}$ & Total \\
\hline $04 / 30 / 93$ & 15 & 8.1 & 28 & 4.9 & 26 & 10 & 36 & 4,690 & 83 & 65 & 0 & 0 & 0 & 4,970 \\
\hline $05 / 27 / 93$ & 16 & 10 & 51 & 4.5 & 10 & 13 & 45 & 6,060 & 92 & 46 & 0 & 0 & 0 & 6,350 \\
\hline $06 / 30 / 93$ & 22 & 11 & 56 & 5.4 & 37 & 8.7 & 40 & 3,490 & 80 & 37 & 0 & 0 & 0 & 3,790 \\
\hline $07 / 30 / 93$ & 26 & 10 & 75 & 9.5 & 11 & 5.3 & 25 & 581 & 38 & 57 & 0 & 0 & 0 & 840 \\
\hline $09 / 30 / 93$ & 17 & 14 & 46 & 6.2 & 34 & 8.9 & 31 & 367 & 87 & 59 & 0 & 0 & 0 & 670 \\
\hline $10 / 22 / 93$ & 8.8 & 13 & 38 & 4.2 & 35 & 6.7 & 30 & 398 & 69 & 59 & 0 & 0 & 0 & 660 \\
\hline $12 / 06 / 93$ & 0 & 0.9 & 0 & 0 & 0 & 0 & 29 & 1,250 & 25 & 0 & 0 & 0 & 0 & 1,300 \\
\hline $01 / 28 / 94$ & 0 & 0 & 0 & 0 & 0 & 0 & 20 & 872 & 23 & 0 & 0 & 0 & 0 & 910 \\
\hline $02 / 28 / 94$ & 0 & 0 & 0 & 0 & 0 & 0 & 18 & 857 & 17 & 0 & 0 & 0 & 0 & 890 \\
\hline $04 / 01 / 94$ & 27 & 7.4 & 32 & 11 & 20 & 8.5 & 35 & 1,460 & 110 & 61 & 0 & 0 & 0 & 1,770 \\
\hline $05 / 02 / 94$ & 16 & 6 & 44 & 2.1 & 9.6 & 7.2 & 35 & 3,100 & 109 & 48 & 0 & 0 & 0 & 3,380 \\
\hline $06 / 27 / 94$ & 20 & 21 & 43 & 5.6 & 12 & 20 & 40 & 4,790 & 91 & 53 & 0 & 0 & 0 & 5,090 \\
\hline $08 / 29 / 94$ & 11 & 14 & 44 & 9.2 & 15 & 12 & 25 & 423 & 91 & 62 & 0 & 0 & 0 & 710 \\
\hline $09 / 30 / 94$ & 15 & 8.8 & 62 & 6 & 34 & 11 & 26 & 329 & 76 & 24 & 0 & 0 & 0 & 590 \\
\hline $10 / 31 / 94$ & 6.7 & 5.4 & 30 & 0 & 20 & 10 & 26 & 396 & 110 & 34 & 0 & 0 & 0 & 640 \\
\hline $12 / 01 / 94$ & 0 & 0 & 0 & 0 & 0 & 0 & 23 & 734 & 22 & 0 & 0 & 0 & 0 & 780 \\
\hline $01 / 30 / 95$ & 0 & 0 & 0 & 0 & 0 & 0 & 21.4 & 1,020 & 24.6 & 0 & 0 & 0 & 0 & 1,070 \\
\hline $02 / 28 / 95$ & 0 & 0 & 0 & 0 & 0 & 0 & 22.1 & 1,630 & 23.5 & 0 & 0 & 0 & 0 & 1,680 \\
\hline
\end{tabular}


Table 4.--Surface-water discharge and differences between Rio Grande discharge and total discharge from the Paseo del Norte (north) section and the Rio Bravo Boulevard (south) section

[See figure 2 for location and table 1 for names of sites. Values are in cubic feet per second;

\begin{tabular}{|c|c|c|c|c|c|c|}
\hline \multirow[b]{2}{*}{ Date } & \multicolumn{3}{|c|}{ Rio Grande discharge } & \multicolumn{3}{|c|}{ Total discharge } \\
\hline & Site 4 & Site 18 & Difference & North section & South section & Difference \\
\hline $\begin{array}{l}08 / 25 / 89 \\
09 / 29 / 89 \\
10 / 27 / 89 \\
12 / 01 / 89 \\
12 / 29 / 89\end{array}$ & $\begin{array}{r}251 \\
22 \\
115 \\
171 \\
757\end{array}$ & $\begin{array}{r}243 \\
21 \\
112 \\
131 \\
729\end{array}$ & $\begin{array}{r}-8 \\
-1 \\
-3 \\
-40 \\
-28\end{array}$ & $\begin{array}{l}600 \\
320 \\
410 \\
390 \\
820\end{array}$ & $\begin{array}{l}530 \\
240 \\
300 \\
360 \\
770\end{array}$ & $\begin{array}{r}-70 \\
-80 \\
-110 \\
-30 \\
-50\end{array}$ \\
\hline $\begin{array}{l}01 / 31 / 90 \\
02 / 28 / 90 \\
03 / 30 / 90 \\
04 / 30 / 90 \\
05 / 31 / 90\end{array}$ & $\begin{array}{r}518 \\
292 \\
875 \\
681 \\
1,190\end{array}$ & $\begin{array}{r}511 \\
545 \\
715 \\
735 \\
1,020\end{array}$ & $\begin{array}{r}-7 \\
253 \\
-160 \\
54 \\
-170\end{array}$ & $\begin{array}{r}580 \\
340 \\
1,180 \\
1,030 \\
1,600\end{array}$ & $\begin{array}{r}560 \\
590 \\
1,020 \\
930 \\
1,330\end{array}$ & $\begin{array}{r}-20 \\
250 \\
-160 \\
-100 \\
-270\end{array}$ \\
\hline $\begin{array}{l}06 / 29 / 90 \\
07 / 31 / 90 \\
08 / 30 / 90 \\
09 / 27 / 90 \\
10 / 30 / 90\end{array}$ & $\begin{array}{l}428 \\
398 \\
285 \\
260 \\
307\end{array}$ & $\begin{array}{l}313 \\
269 \\
183 \\
296 \\
360\end{array}$ & $\begin{array}{r}-115 \\
-129 \\
-102 \\
36 \\
53\end{array}$ & $\begin{array}{l}730 \\
720 \\
620 \\
660 \\
630\end{array}$ & $\begin{array}{l}580 \\
540 \\
510 \\
570 \\
580\end{array}$ & $\begin{array}{r}-150 \\
-180 \\
-110 \\
-90 \\
-50\end{array}$ \\
\hline $\begin{array}{l}12 / 11 / 90 \\
01 / 10 / 91 \\
01 / 31 / 91 \\
02 / 21 / 91 \\
03 / 27 / 91\end{array}$ & $\begin{array}{r}699 \\
1,040 \\
742 \\
869 \\
1,290\end{array}$ & $\begin{array}{r}652 \\
1,060 \\
737 \\
898 \\
1,450\end{array}$ & $\begin{array}{r}-47 \\
20 \\
-5 \\
29 \\
160\end{array}$ & $\begin{array}{r}770 \\
1,100 \\
810 \\
930 \\
1,610\end{array}$ & $\begin{array}{r}710 \\
1,110 \\
780 \\
950 \\
1,680\end{array}$ & $\begin{array}{r}-60 \\
10 \\
-30 \\
20 \\
70\end{array}$ \\
\hline $\begin{array}{l}05 / 02 / 91 \\
05 / 30 / 91 \\
06 / 28 / 91 \\
07 / 31 / 91 \\
08 / 29 / 91\end{array}$ & $\begin{array}{r}3,580 \\
3,920 \\
3,490 \\
953 \\
399\end{array}$ & $\begin{array}{r}2,970 \\
4,090 \\
3,050 \\
1,150 \\
270\end{array}$ & $\begin{array}{r}-610 \\
170 \\
-440 \\
197 \\
-129\end{array}$ & $\begin{array}{r}3,980 \\
4,340 \\
3,840 \\
1,350 \\
770\end{array}$ & $\begin{array}{r}3,250 \\
4,440 \\
3,370 \\
1,520 \\
580\end{array}$ & $\begin{array}{r}-730 \\
100 \\
-470 \\
170 \\
-190\end{array}$ \\
\hline $\begin{array}{l}09 / 26 / 91 \\
10 / 31 / 91 \\
11 / 27 / 91 \\
12 / 31 / 91 \\
01 / 30 / 92\end{array}$ & $\begin{array}{r}645 \\
320 \\
1,430 \\
1,050 \\
859\end{array}$ & $\begin{array}{r}600 \\
267 \\
1,270 \\
913 \\
824\end{array}$ & $\begin{array}{r}-45 \\
-53 \\
-160 \\
-137 \\
-35\end{array}$ & $\begin{array}{r}-- \\
660 \\
1,490 \\
1,110 \\
910\end{array}$ & $\begin{array}{r}840 \\
560 \\
1,330 \\
960 \\
880\end{array}$ & $\begin{array}{r}-- \\
-100 \\
-160 \\
-150 \\
-30\end{array}$ \\
\hline $\begin{array}{l}02 / 19 / 92 \\
04 / 08 / 92 \\
04 / 30 / 92 \\
06 / 30 / 92 \\
07 / 31 / 92\end{array}$ & $\begin{array}{r}306 \\
2,890 \\
5,560 \\
912 \\
734\end{array}$ & $\begin{array}{r}338 \\
2,640 \\
5,120 \\
815 \\
687\end{array}$ & $\begin{array}{r}32 \\
-250 \\
-440 \\
-97 \\
-47\end{array}$ & $\begin{array}{r}360 \\
3,210 \\
5,920 \\
1,220 \\
1,120\end{array}$ & $\begin{array}{r}-- \\
2,960 \\
5,470 \\
1,090 \\
1,010\end{array}$ & $\begin{array}{r}-- \\
-250 \\
-450 \\
-130 \\
-110\end{array}$ \\
\hline $\begin{array}{l}08 / 31 / 92 \\
09 / 30 / 92 \\
11 / 02 / 92 \\
11 / 30 / 92 \\
01 / 04 / 93\end{array}$ & $\begin{array}{r}1,120 \\
566 \\
608 \\
614 \\
1,160\end{array}$ & $\begin{array}{r}1,260 \\
543 \\
501 \\
690 \\
1,060\end{array}$ & $\begin{array}{r}140 \\
-23 \\
-107 \\
76 \\
-100\end{array}$ & $\begin{array}{r}1,490 \\
940 \\
730 \\
680 \\
1,220\end{array}$ & $\begin{array}{r}1,550 \\
800 \\
600 \\
730 \\
1,110\end{array}$ & $\begin{array}{r}60 \\
-140 \\
-130 \\
50 \\
-110\end{array}$ \\
\hline $\begin{array}{l}01 / 28 / 93 \\
02 / 26 / 93 \\
03 / 31 / 93 \\
04 / 30 / 93 \\
05 / 27 / 93\end{array}$ & $\begin{array}{l}1,030 \\
1,940 \\
2,840 \\
4,880 \\
5,900\end{array}$ & $\begin{array}{l}1,070 \\
2,460 \\
2,830 \\
4,690 \\
6,060\end{array}$ & $\begin{array}{r}40 \\
520 \\
-10 \\
-190 \\
160\end{array}$ & $\begin{array}{l}1,120 \\
2,010 \\
3,150 \\
5,170 \\
6,310\end{array}$ & $\begin{array}{l}1,110 \\
2,500 \\
3,100 \\
4,970 \\
6,350\end{array}$ & $\begin{array}{r}-10 \\
490 \\
-50 \\
-200 \\
40\end{array}$ \\
\hline
\end{tabular}


Table 4.--Surface-water discharge and differences between Rio Grande discharge and total discharge from the Paseo del Norte (north) section and the Rio Bravo Boulevard (south) section-Concluded

\begin{tabular}{crrrrrrrr}
\hline & \multicolumn{3}{c}{ Rio Grande discharge } & & \multicolumn{3}{c}{ Total discharge } \\
\cline { 2 - 3 } \cline { 7 - 8 } Date & Site 4 & Site 18 & Difference & & North section & South section & Difference \\
\hline $06 / 30 / 93$ & 3,550 & 3,490 & -60 & & 3,920 & 3,790 & -130 \\
$07 / 30 / 93$ & 811 & 581 & -230 & & 1,110 & 840 & -270 \\
$09 / 30 / 93$ & 438 & 367 & -71 & & 730 & 670 & -60 \\
$10 / 22 / 93$ & 450 & 398 & -52 & & 740 & 660 & -80 \\
$12 / 06 / 93$ & 1,420 & 1,250 & -170 & & 1,490 & 1,300 & -190 \\
$01 / 28 / 94$ & 943 & 872 & -71 & & 1,000 & 910 & -90 \\
$02 / 28 / 94$ & 1,040 & 857 & -183 & & 1,090 & 890 & -200 \\
$04 / 01 / 94$ & 1,470 & 1,460 & -10 & & 1,790 & 1,770 & -20 \\
$05 / 02 / 94$ & 3,230 & 3,100 & -130 & & 3,580 & 3,380 & -200 \\
$06 / 27 / 94$ & 5,300 & 4,790 & -510 & & 5,630 & 5,090 & -540 \\
$08 / 29 / 94$ & 510 & 423 & -87 & & 830 & 710 & -120 \\
$09 / 30 / 94$ & 375 & 329 & -46 & & 760 & -170 \\
$10 / 31 / 94$ & 397 & 396 & -1 & & 700 & 640 & -60 \\
$12 / 01 / 94$ & 783 & 734 & -49 & & 860 & 780 & -80 \\
$01 / 30 / 95$ & 1,040 & 1,020 & -20 & & 1,090 & 1,070 & -20 \\
$02 / 28 / 95$ & 1,610 & 1,630 & 20 & & 1,670 & 1,680 & 10 \\
\hline
\end{tabular}




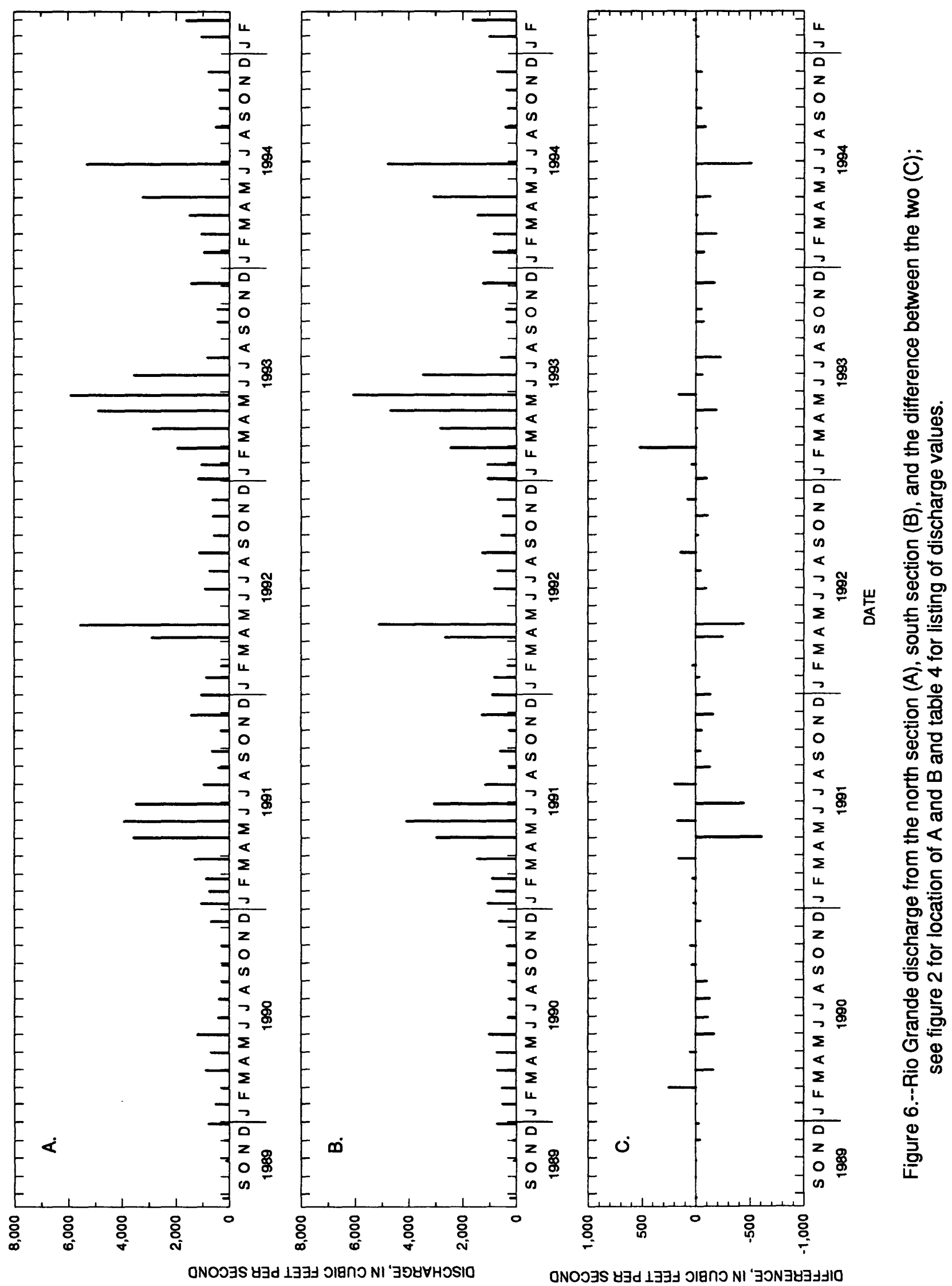




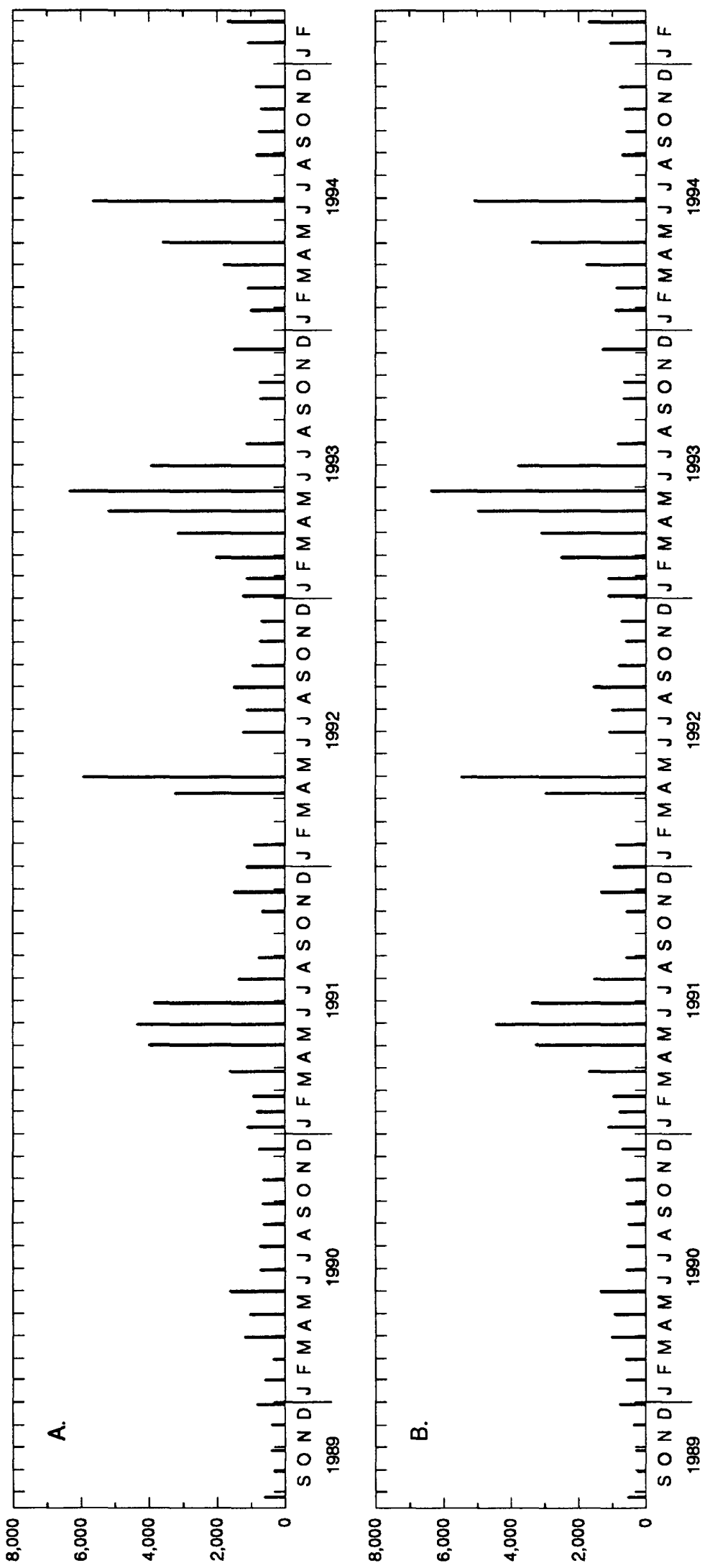

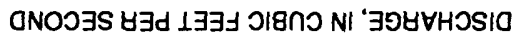

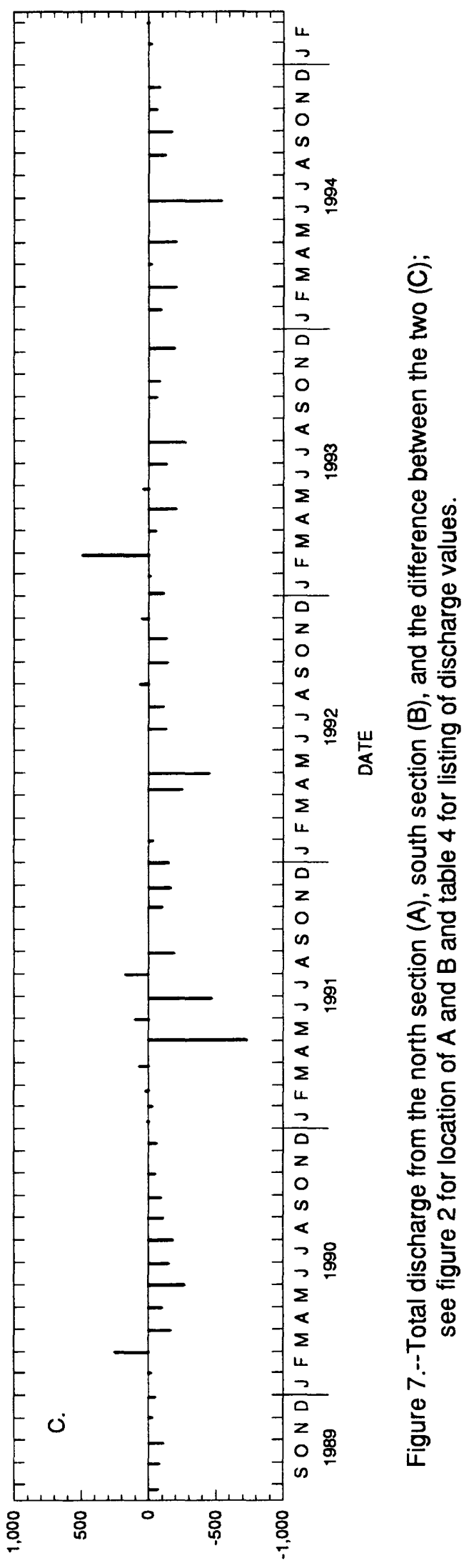

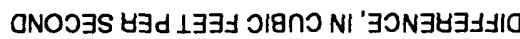




\section{EVAPOTRANSPIRATION RATES}

The evapotranspiration rates from six areas located at Paseo del Norte and the Rio Grande, between surface-water measurement sites 4 and 5 (fig. 2), ranged from 0.13 to 6.4 millimeters per day (table 5). Three areas are covered with grass and three areas are bare soil (fig. 8). These areas represent the evapotranspiration contribution of grass-covered and bare soil along the edge of the river. All six areas are located on the east side of the river along a line perpendicular to the river. Area 1 is closest to the river, 20 to 100 feet from the river edge, depending on the stage of the river at the time of data collection. Area 6, located the farthest distance from the river, is 700 to 800 feet from the river edge. Data were collected from February 23, 1990, through August 29, 1991 (table 5 and fig. 9).

Data were collected using a hemispherical chamber placed at each sampling area for 2minute intervals (fig. 8). Wet- and dry-bulb temperatures were measured with a psychrometer mounted on the inside of the chamber. Two fans were also mounted on the inside of the chamber to create optimal air mixing during the measurement interval (fig. 8). The wet- and dry-bulb temperatures were recorded every 2 seconds during the 2 minutes the chamber was on the sampling site. A Campbell 21X micrologger ${ }^{1}$ was used to record the date, time of day, and wetand dry-bulb temperatures. The sampling effort always began at area 1 and consecutively progressed to area 6 . At the start of each area visit the fans mounted on the inside of the chamber were turned on for 10 to 15 seconds while holding the chamber 3 to 4 feet above ground. The data logger was then turned on and the chamber placed onto the ground, ensuring a proper seal between the ground and the rim of the chamber. At the end of the 2-minute interval the data logger was switched off and the chamber carried to the next area. Once all six areas were visited, 20 minutes would have elapsed before area 1 was revisited for the start of the second round of area visits. This sequence of area visits took place from sunrise to sundown.

From the wet- and dry-bulb temperature data, vapor density curves were constructed and evapotranspiration rates for each area were calculated (table 5). For a complete understanding of data collection using the hemispherical chamber and calculation of evapotranspiration rates, the reader is referred to Stannard (1988). Generally, the greatest evapotranspiration rates are associated with the bare-soil conditions of area 1 (table 5). This area is closest to the river and the evapotranspiration rates are affected by an increase of soil moisture from the river--that is, the site was moist during most visits. Evapotranspiration rates from areas 5 and 6 are shown in figure 9. These two areas were selected to graphically compare the evapotranspiration rates between bare-soil and grass-covered areas that are farthest from the river and least affected by soil moisture contributed from the river. The grass-covered area generally has a greater evapotranspiration rate than the bare-soil area (fig. 9). The exception to this occurred on September 27, 1990; an improper seal between the ground surface and chamber could explain this exception.

${ }^{1}$ Use of brand names in this report is for identification purposes only and does not constitute endorsement by the U.S. Geological Survey. 
Table 5.--Evapotranspiration rates at six areas at Paseo del Norte and the Rio Grande, February 1990 through August 1991

[Area 1: bare soil, moist; area 2: grass-covered, moist soil; areas 3 and 5: bare soil, dry; areas 4 and 6: grass-covered, dry soil. All rates are in millimeters per day]

\begin{tabular}{lllllll}
\hline Date & Area 1 & Area 2 & Area 3 & Area 4 & Area 5 & Area 6 \\
\hline & & & & & & \\
$02 / 23 / 90$ & 1.3 & 0.96 & 0.50 & 0.59 & 0.73 & 0.85 \\
$05 / 17 / 90$ & 4.3 & 3.8 & 1.9 & 2.2 & 2.5 & 2.7 \\
$06 / 28 / 90$ & 6.4 & 4.2 & 2.8 & 3.2 & 2.5 & 2.8 \\
$07 / 26 / 90$ & 6.1 & 4.7 & 0.78 & 2.3 & 1.3 & 2.8 \\
$08 / 30 / 90$ & 6.3 & 1.2 & 0.65 & 1.1 & 1.2 & 1.6 \\
& & & & & & \\
$09 / 27 / 90$ & 3.5 & 0.60 & 0.29 & 0.53 & 0.32 & 0.16 \\
$10 / 25 / 90$ & 1.8 & 0.59 & 0.14 & 0.21 & 0.15 & 0.22 \\
$11 / 29 / 90$ & 0.27 & 0.31 & 0.17 & 0.16 & 0.13 & 0.15 \\
$01 / 17 / 91$ & 0.57 & 0.55 & 0.14 & 0.23 & 0.17 & 0.24 \\
$03 / 14 / 91$ & 1.2 & 0.20 & 0.14 & 0.21 & 0.14 & 0.18 \\
$04 / 25 / 91$ & 1.7 & 1.9 & 0.17 & 0.50 & 0.20 & 0.63 \\
$05 / 23 / 91$ & 4.6 & 4.2 & 1.0 & 1.1 & 0.82 & 1.5 \\
$06 / 27 / 91$ & 1.6 & 4.3 & 0.31 & 1.3 & 0.29 & 0.51 \\
$08 / 01 / 91$ & 0.52 & 3.0 & 0.44 & 1.3 & 0.43 & 1.5 \\
$08 / 29 / 91$ & 0.62 & 1.4 & 0.54 & 0.91 & 0.37 & 0.82 \\
\hline
\end{tabular}


A.

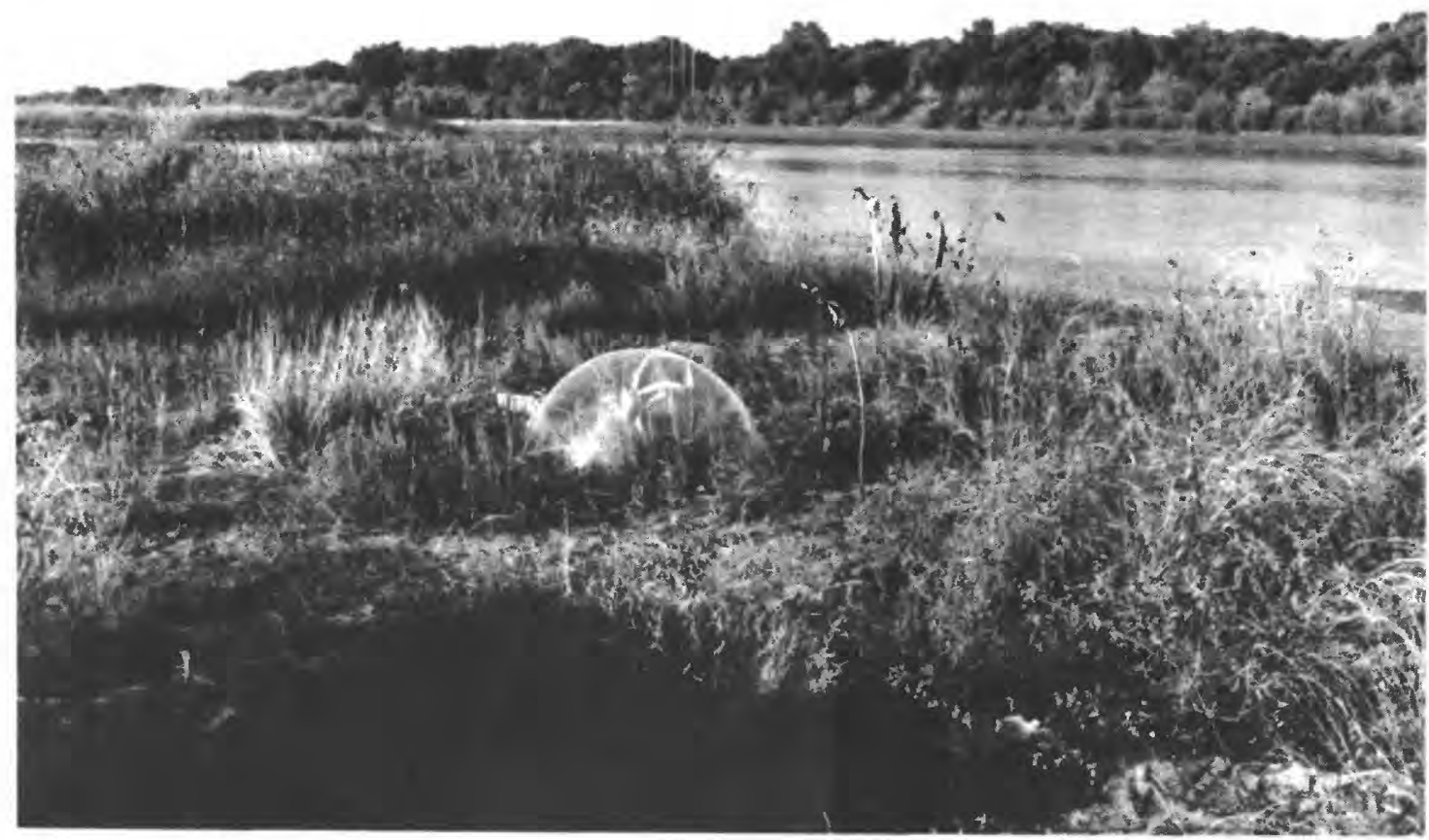

B.

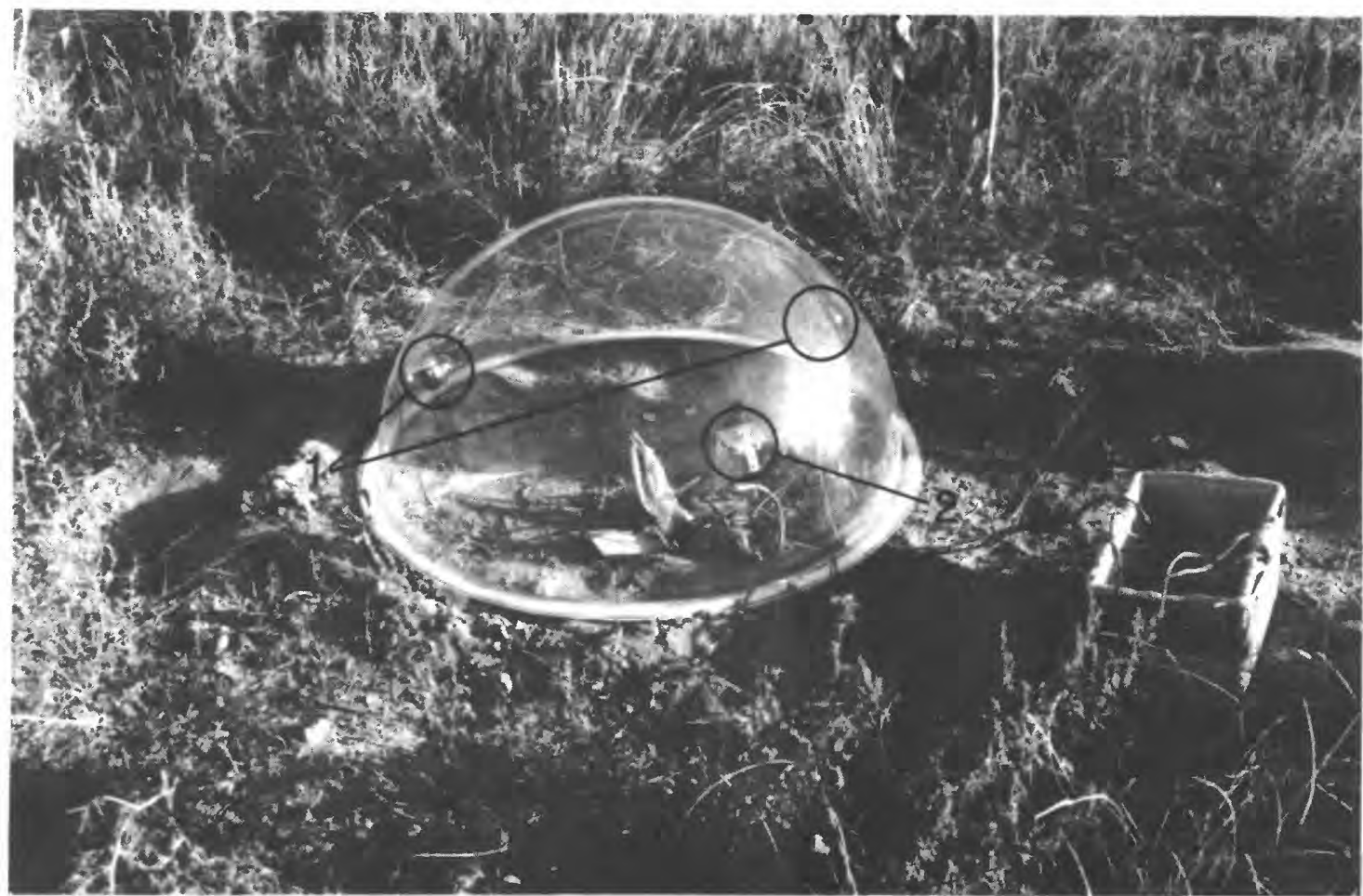

Figure 8.--Hemispherical chamber located at Paseo del Norte and the Rio Grande: (A) grass covered (area 2); (B) bare soil (area 3). Note location of fans (1) and psychrometer (2) mounted on inside of chamber. Also shown in $B$ is the data logger to the right of the chamber. 


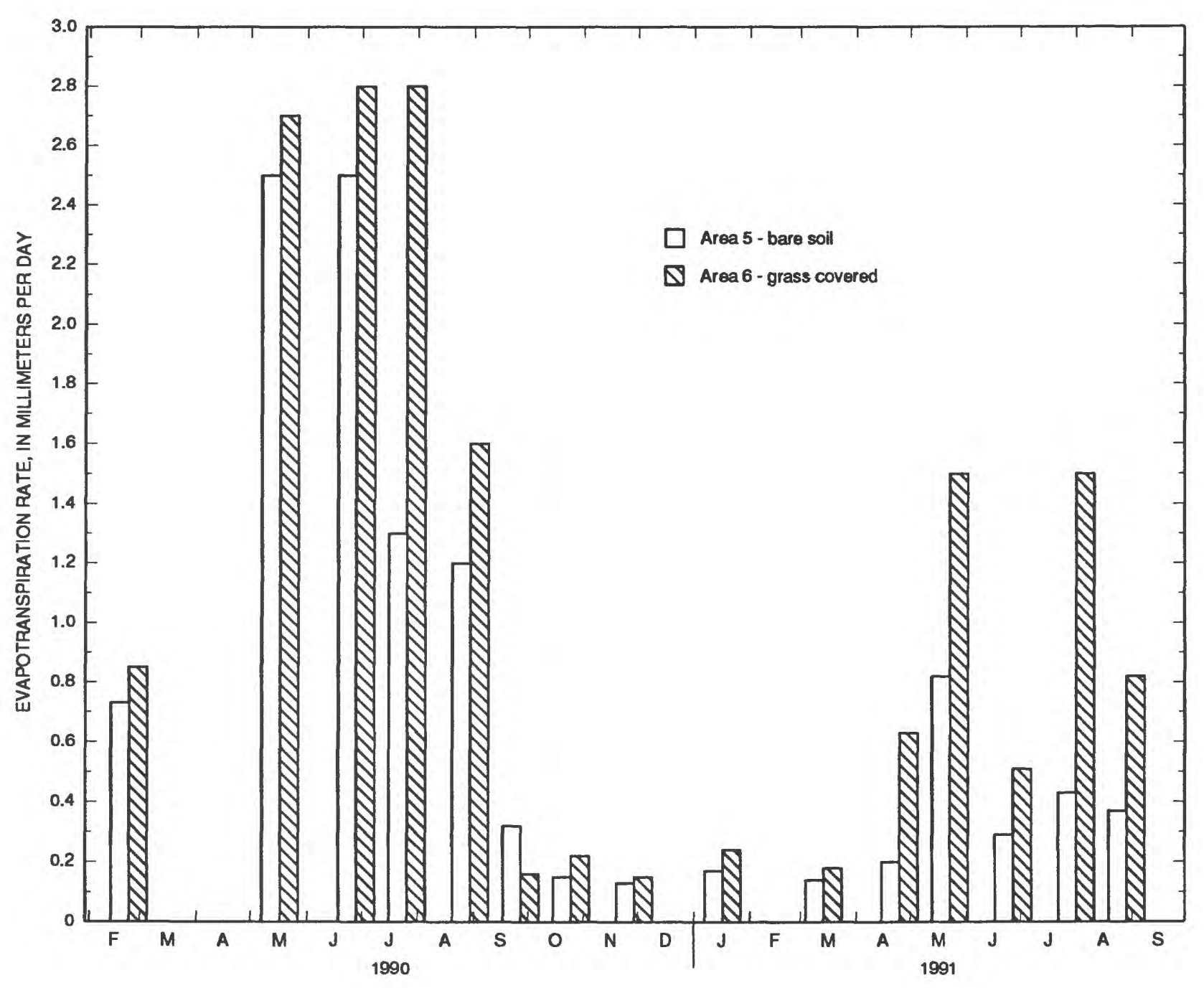

Figure 9.--Evaporanspiration rates calculated at areas 5 and 6 at Paseo del Norte and Rio Grande (location shown in figure 2). 


\section{SELECTED REFERENCES}

Borland, J.P., Cruz, R.R., McCracken, R.L., Lepp, R.L., Ortiz, D., and Shaull, D.A., 1991, Water resources data, New Mexico, water year 1990: U.S. Geological Survey Water-Data Report NM-90-1, $466 \mathrm{p}$.

Borland, J.P., DeWees, R.K., McCracken, R.L., Lepp, R.L., Ortiz, D., and Shaull, D.A., 1990, Water resources data, New Mexico, water year 1989: U.S. Geological Survey Water-Data Report NM-89-1, 426 p.

1992, Water resources data, New Mexico, water year 1991: U.S. Geological Survey WaterData Report NM-91-1, 557 p.

Borland, J.P., and Ong, Kim, 1995, Water resources data, New Mexico, water year 1994: U.S. Geological Survey Water-Data Report NM-94-1, 581 p.

Buchanan, T.J., and Somers, W.P., 1984, Discharge measurements at gaging stations: Techniques of Water-Resources Investigations of the U.S. Geological Survey, book 3, chap. A8, 65 p.

Carter, R.W., and Davidian, J., 1989, General procedure for gaging streams: Techniques of WaterResources Investigations of the U.S. Geological Survey, book 3, chap. A6, 13 p.

Crawford, C.S., Cully, A.C., Leutheuser, R., Sifuentes, M.S., White, L.H., and Wilber, J.P., 1993, Middle Rio Grande ecosystem--bosque biological management plan: Biological Interagency Team, U.S. Fish and Wildlife Service, Albuquerque, N. Mex., 291 p.

Cruz, R.R., DeWees, R.K., Funderburg, D.E., Lepp, R.L., Ortiz, D., and Shaull, D., 1993, Water resources data, New Mexico, water year 1992: U.S. Geological Survey Water-Data Report NM-92-1, 526 p.

1994, Water resources data, New Mexico, water year 1993: U.S. Geological Survey WaterData Report NM-93-1, 590 p.

Kennedy, E.J., 1984, Discharge ratings at gaging stations: Techniques of Water-Resources Investigations of the U.S. Geological Survey, book 3, chap. A10, 59 p.

Kernodle, J.M., Miller, R.S., and Scott, W.B., 1987, Three-dimensional model simulation of transient ground-water flow in the Albuquerque-Belen Basin, New Mexico: U.S. Geological Survey Water-Resources Investigations Report 86-4194, $86 \mathrm{p}$.

Stannard, D.I., 1988, Use of a hemispherical chamber for the measurement of evapotranspiration: U.S. Geological Survey Open-File Report 88-452, 18 p.

Thorn, C.R., McAda, D.P., and Kernodle, J.M., 1993, Geohydrologic framework and hydrologic conditions in the Albuquerque Basin, Central New Mexico: U.S. Geological Survey WaterResources Investigations Report 93-4149, 106 p. 